\title{
A Decision Support System for Preventing Falls in Elderly People
}

\author{
Estelle Courtial \\ Laboratory PRISME, \\ University of Orléans \\ 8 rue Léonard de Vinci \\ 45072 Orléans, France \\ Estelle.Courtial@univ- \\ orleans.fr
}

\author{
Damien Brulin \\ LAAS-CNRS, Université de \\ Toulouse, UT2J \\ 7 avenue du Colonel Roche \\ F-31400 Toulouse, France \\ dbrulin@laas.fr
}

\author{
Caroline Barelle \\ Laboratory PRISME, \\ University of Orléans \\ 8 rue Léonard de Vinci \\ 45072 Orléans, France \\ caroline.barelle@univ- \\ orleans.fr
}

\begin{abstract}
Preventing falls in older people is a real challenge for Public Health. This paper addresses this issue by designing a decision support system which provides a fall risk index. The proposed approach is based on three selected tests (the Timed up and go (TUG), the 30s sit-to-stand and the 4stage balance tests), which are widely used in the medical sector for assessing mobility and balance of the elderly. During the tests, a video records the older person performing the test and thanks to an image processing algorithm, kinematics and biomechanics parameters are extracted. Based on fuzzy logic, a decision support system fuses all these data and estimates a fall risk index according to the senior's age and gender. It can also assist the Health Professional in making improved medical diagnosis relied on targeted measurements. Simulation results drawing on experimental data of 12 older persons performing the TUG test illustrate the feasibility and the effectiveness of the proposed approach. Objectively assessing the senior's motor functions and the fall risk is possible in less than 10 minutes, at low cost and in an easy and non invasive way.
\end{abstract}

\section{Categories and Subject Descriptors}

B.1.2 [Control Structure Performance Analysis and Design Aids]: Automatic synthesis

\section{General Terms}

Theory, Algorithms

\section{INTRODUCTION}

United Nations [6] reports that the world's population aged 65 years and over will increase from 620 million in 2010 to 1.5 billion in 2050 , representing $16 \%$ of the global population (5\% in 1950). The demographic ageing of the population is

\footnotetext{
* corresponding author
}

mainly due to the increase of life expectancy at birth. In the last five decades, people live longer by almost 20 years globally, with an average of 66.6 years. The proportion of old persons aged of 80 and over is more and more important in the older population.

According to the World Health Organization (WHO) [12], the fall is the second leading cause of death worldwide among the adults over 65 years old, and the first cause among the adults over 80 years old. Approximately $30 \%$ of the elderly (over 65 years) fall each year. Fall, if not deadly, results in physical injuries (often inducing hospitalization, loss of independence) and also in psychological damages (loss of confidence, fear of falling, low self-esteem) leading to depression and social isolation. The direct and indirect costs related to falls consume a major part of the Health budget. Preventing falls is thus a real Health challenge with economic and societal issues. Public Health actions recommended by the WHO focus on the improvement of identification and assessment of risk factors. In this context, our study specifically deals with the assessment of the fall risk in a preventive strategy.

From a medical point of view, current methods for assessing gait and balance impairments are Tinetti Gait and Balance tests, Berg balance, sit-to-stand and Timed up and go tests [8], [2]. These tests are often driven by a subjective observation of the patient by a Health Professional (HP), a physician or a physiotherapist. The assessment is thus subject to variations in results.

During the last decade, the gait analysis and the gait evolution associated with the ageing process have largely been investigated in the literature [9],[10]. Most of the kinematics and biomechanics parameters characterizing the motor functions can be collected, in lab or in everyday life, from different technological means such as instrumented surfaces (e.g gait analysis mat,GAITRite (B), force platform, treadmill, smart soles in shoes [3], magneto-inertial sensors worn by the elderly [11], video-based system (VICON system (B) [7], etc. These solutions are either high cost, or non-transportable, or non-secure for elderly, or require time to be carried out (sensors installation and calibration). Furthermore, existing solutions generally extract only the relevant parameters but do not analyze the data and let the HP evaluating the patient's fall risk from information gathered during the tests. In this study, we address this issue by designing a complete solution from data extraction to a decision support system (DSS) which provides a fall risk index (FRI). Preventing falls 
can mainly rely on four criteria: the gait, the balance, the flexibility and the strength. We consider three tests among the current clinical screening tests for assessing mobility and balance impairments: the Timed up and go, the 4-stage balance and the 30s sit-to-stand tests. Their combination can be considered as an efficient tool for identifying the fall risk in older people. For the practical implementation we opt for a video-based technology (a single optical sensor) which is low-cost, readily available, non-invasive and meet the end-users' requirements in terms of ease-of-use and userfriendliness. Then an image processing algorithm extracts all the relevant parameters corresponding to the inputs of the DSS. The latter provides a fall risk index by fusing the different measurements.

The remainder of this paper is organized as follows. In Section II, the four criteria for fall prevention and the three selected tests are detailed. Section III is devoted to the image processing algorithm. In section IV, the DSS is developed. In Section V, simulation results illustrate the feasibility and the effectiveness of the proposed approach. Finally, conclusions and perspectives are presented in Section VI.

\section{ASSESSMENT TESTS FOR GAIT AND BALANCE}

A normal gait simultaneously requires a satisfactory balance, a certain range of joint flexibility (hip, knee, ankle) and a sufficient muscle strength to perform the motion. Preventing falls can rely on the assessment of four criteria (GBFS): The Gait (G) whom cycle is made up of stance and swing phases. It is usually characterized by spatio-temporal parameters [11] such as the step length, distance between the heel contact point of one foot and the heel contact point of the other foot, the gait velocity and the step frequency.

The Balance (B) is the capacity for a person to feel where the different parts of his/her body are located in the space as well as the capacity to maintain a desired position. It depends on information from the inner ear (labyrinth), the sight, the touch and the muscular strength.

The Flexibility (F) is the ability of the joint (the muscular tissue makes possible the elongation) to perform the optimal amplitude of movement. Significant reduction of this property may compromise the suitable execution of the movement. Flexibility depends on joint structure, ligaments and muscles.

The Strength (S) is the amount of force generated by the muscle contraction against a resistance.

In order to assess these criteria (GBFS), we selected three well-known tests widely used in the medical sector:

The Timed Up and Go (TUG) test is a screening test for gait impairments [8], [5]. It measures basic motor functions including functional actions used in the activities of daily life (to turn, to sit, to stand). The TUG test consists in standing up from a chair, walking 3 meters, turning around a spot, walking back to the chair and sitting down (see Fig. 1). The HP usually records the time required to perform the test and classifies the patient's locomotive status into normal or impaired. The cut-off value is generally set to approximatively $12-14$ s according to the age.

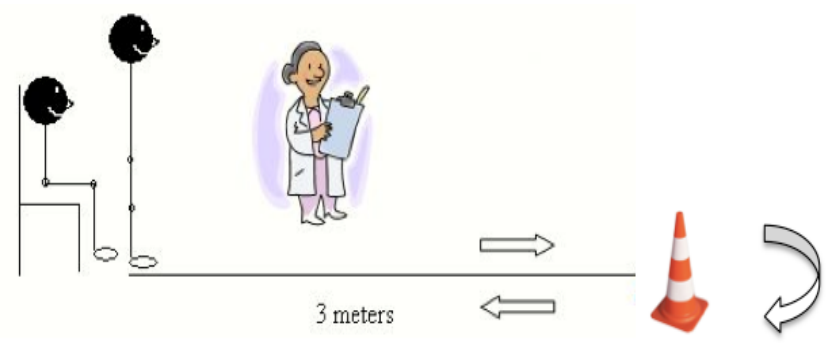

Figure 1: The Timed up and go test.

The 30s sit-to-stand test: the patient is sitting on a chair with their hands on the opposite shoulders, crossed at the wrists. During 30 seconds the patient should repeat the movement "stand up and sit down" while keeping their arms against their chest.

The 4-stage balance test is focused on the static balance assessment (see Fig. 2). The patient should maintain each position at least 10 seconds while keeping their eyes open.

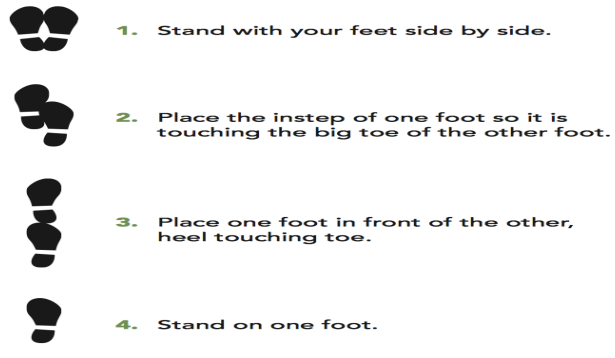

Figure 2: The 4-stage balance test.

During the TUG test and thanks to the image processing algorithm (detailed in the next section), spatio-temporal parameters can be extracted as well as the active range of motion (AROM) of the joints, respectively assessing the gait and the flexibility. The balance (dynamic and static) is evaluated with time measurements during the TUG and 4-stage balance tests. The strength is estimated according to the number of sit-to-stand performed in 30 seconds [2].

\section{IMAGE PROCESSING}

The developed image processing method is divided into 2 modules: a detection and tracking module and a gait feature extraction module (see Fig. 3). Motion analysis based on video has been largely investigated in the literature for biomechanics studies. Methods using markers on the body, specially with the advanced VICON system [7] were widely applied for gait analysis. However these methods are not adapted to a screening test which requires to be as less invasive as possible for the patient, quickly performed and easy to implement. Recently automated markerless methods for gait analysis were developed [4]. In the same spirit, we combine classical image processing methods with anatomical considerations leading to a markerless method fulfilling requirements in terms of accuracy and low computation load.

\subsection{Detection and tracking module}

The operating conditions are the following:

(1) the video is recording thanks to a fixed single monocular 


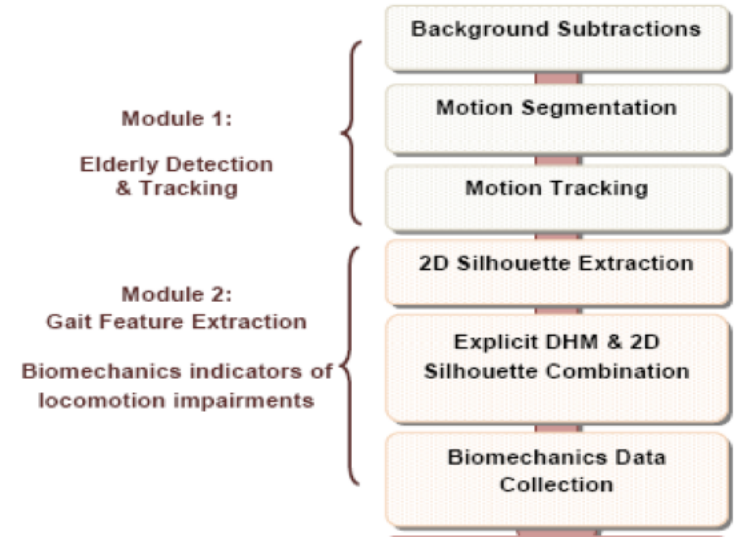

Figure 3: Schema of the image processing method.

network camera, at a sufficient distance from the old person in order to acquire the side views of the senior's walking (distance $1.5 \mathrm{~m}$ height $1.65 \mathrm{~m}$ );

(2) the test is performed in an indoor environment with low variable lighting conditions;

(3) in order to respect privacy and ethical issues, the silhouette of the elderly is stored solely in black and white.

For this first module, a classical approach is used. The video stream is transformed into a series of frames. A background subtraction is performed for each frame of the video sequence. The next step consists of detecting the edge in the image by using the Prewitt gradient magnitude method, i.e. returning edges at those points where the gradient of the image is maximum, especially at image locations representing object boundaries. Finally, an enhancement procedure based on morphological operators is performed on the resulting image for removing noise as much as possible. This operation adding to a connected component process is crucial for an accurate contour and a compact connected region extraction of the walking figure (see Fig. 4). In this study, tracking is carried out in a easy way since the only moving object is the older person walking.

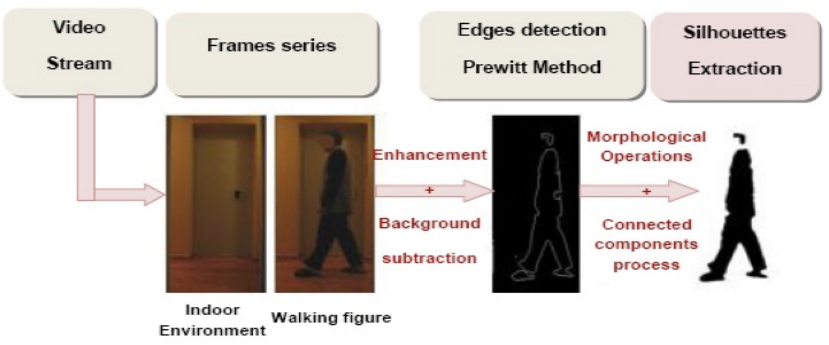

Figure 4: steps of the image processing method

\subsection{Gait feature extraction module}

This module aims at extracting the spatio-temporal gait parameters and the temporal evolution of the skeletal articulation structure of the person. The $2 \mathrm{D}$ coordinates of all the pixels on the outer contour of the silhouette (border following algorithm) are raised. Then the coordinates of nine points on the body (head, neck, shoulder, torso, pelvis, 2 knees, 2 ankles) are extracted by topological analysis and linear regression guided by anatomical knowledge as proposed in [14] (see Fig. 5 ). The vertical positions of the

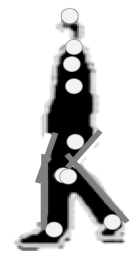

Figure 5: The nine points (in white) and linear regressions (grey line).

9 points are estimated thanks to the model proposed by [13] and the knowledge of the person's height. By following the position of these 9 points on each silhouette frame of the video stream, spatio-temporal gait parameters as well as the joint AROM are extracted or calculated in an easy way [1]. All these values will be the inputs of the DSS.

\section{DSS FOR FALL PREVENTION}

The overall DSS scheme is described in the following figure (see Fig. 6 ). The DSS is based on fuzzy logic. Fuzzy

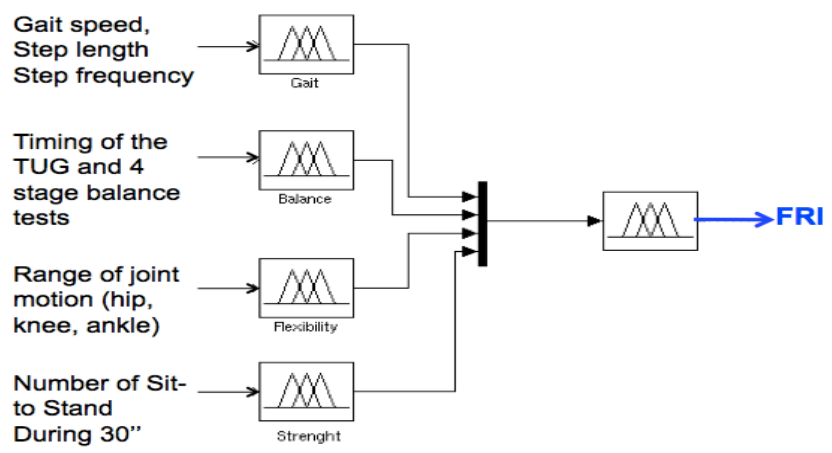

Figure 6: Schema of the DSS.

logic makes possible to match inputs to outputs whatever the relationship (linear or nonlinear) between them and is also well adapted to deal with imprecise data. The design of a Fuzzy Logic System (FLS) requires three steps:

\section{The Fuzzification:}

Fuzzy subsets are identified and defined for each input or output. The number of fuzzy subsets depends on the sensitivity of each input with regard to the output, the fall risk index (FRI). Indeed, two subsets are defined for the flexibility parameters (hip, knee, ankle AROM) while three subsets are defined for the gait parameters. Then the membership function describes the degree of membership (between 0 and 1) of a variable in a subset. It is a curve that defines how a point in the input space is mapped to a membership value. Different types of membership functions exist: triangular, trapezoidal, gaussian or polynomial curves. The choose of the membership function depends on the knowledge and the precision of each input. 
The Fuzzy rules:

The fuzzy rules are structured in an IF-THEN format. For example: IF (the step length is low) and (the gait velocity is low) THEN (the FRI is high). To obtain the membership function solution, we use the aggregation operator max to combine the membership degrees of all rules.

\section{The Defuzzification:}

The final step consists in turning the membership function solution generated by the fuzzy rules into a more comprehensive value. We have chosen the center of gravity method as it is commonly recognized to provide an accurate result of the output membership function.

\section{SIMULATION RESULTS}

\subsection{Data collection}

Two groups of 6 healthy and voluntary older adults made up for equal proportion of women and men took part in the experiment. The first group was composed of 6 persons $(69( \pm 3)$ years $)$ who had not fell in the preceding six months and were able to walk without assistance. The second group comprised 6 older persons $(68( \pm 4)$ years $)$ who had fell at least one time in the last previous months and for whom the fall risk had an impact on their activity of daily living.

We only present here the results obtained in the case of the TUG test. The image processing algorithm provides the spatio-temporal gait parameters (stride length $(\mathrm{m})$, the stride frequency (strides/s), the step length (m), the step frequency (steps/s), the gait cycle duration (s) and the gait speed $(\mathrm{m} / \mathrm{s})$ ) as well as the joint (hip, knee, ankle) AROM. Among them, we decided to use the last seven parameters as inputs of the DSS.

\subsection{Diagnostic of fall risk}

Membership functions have been defined by taking into account the referenced values and their standard deviations given in the literature according to the age and the gender [10] (see Fig. 7). We opted for smooth gaussian membership functions. The position of the center of the peak is equal to the reference value, the width of the curve is equal to the standard deviation associated to the reference value. The FRI output was described by four triangular membership functions on four fuzzy subsets (low risk, moderate risk, risk, high risk). Statistic analysis on experimental data pointed out that the step length and the gait velocity were discriminant factors for the fall risk. Thus these parameters were weighted in the inference system. The rules considered for the TUG test are described in Table 1.

\begin{tabular}{|c|c|c|c|c|c|c|}
\hline \multirow{2}{*}{ Age } & \multicolumn{2}{|c|}{ Gait speed } & \multicolumn{2}{c|}{ Step frequency } & \multicolumn{2}{c|}{ Step length } \\
\cline { 2 - 7 } & Men & Women & Men & Women & Men & Women \\
$($ Years $)$ & $(\mathrm{m} / \mathrm{s})$ & $(\mathrm{m} / \mathrm{s})$ & $($ Steps/s) & $($ Steps/s $)$ & $(\mathrm{m})$ & $(\mathrm{m})$ \\
\hline $50-59$ & $1.252 \pm 0.177$ & $1.105 \pm 0.97$ & $1.96 \pm 0.18$ & $2.03 \pm 0.13$ & $0.635 \pm 0.06$ & $0.535 \pm 0.026$ \\
\hline $60-69$ & $1.277 \pm 0.124$ & $1.157 \pm 0.167$ & $1.95 \pm 0.14$ & $2.06 \pm 0.18$ & $0.65 \pm 0.036$ & $0.553 \pm 0.042$ \\
\hline $70-79$ & $1.182 \pm 0.154$ & $1.113 \pm 0.125$ & $1.91 \pm 0.14$ & $2.03 \pm 0.14$ & $0.615 \pm 0.051$ & $0.542 \pm 0.037$ \\
\hline
\end{tabular}

Figure 7: Referenced norms.

Results are displayed on a Graphical User Interface in different formats (video, text and numerical information) (see Fig. 8). The GUI meets the end-user's requirements in terms of usability and user-friendliness. The HP would like
Table 2: Results of the diagnostic tool

\begin{tabular}{|c|c|c|c|c|}
\hline Group & \multicolumn{4}{|c|}{ Classification } \\
\hline & Low & Moderate & Risk & High \\
\hline elderly (no fallers) & 3 & 2 & 1 & 0 \\
\hline elderly (fallers) & 0 & 1 & 3 & 2 \\
\hline
\end{tabular}

to be able to modify the fall risk index if needed, to replay the video and to add comments. All the data gathered during the tests are stored in a medical database. Results are displayed on a screen in different formats: video, text, numerical information and graphs are available.

The obtained results described in Tab. 2 are consistent to $85 \%$ with those of medical experts and prove the feasibility and reliability of such a tool. The diagnostic was wrong for two persons of small size. Indeed, the step length was short and since we give importance to this factor in the FRI estimation, the two persons were estimated with a risk of fall. The rules and the membership functions have to be refined according to the experience and expertise of the HP.

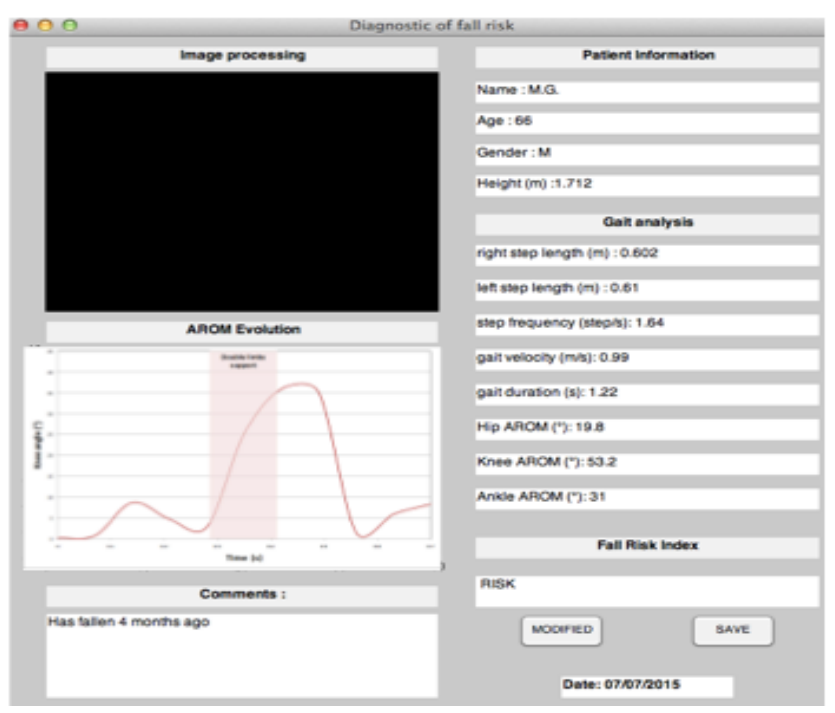

Figure 8: Fuzzy logic module.

\section{CONCLUSIONS}

We developed a diagnostic tool for assessing the elderly's fall risk. For this purpose, an image processing algorithm extracts the relevant parameters during the tests. In regard to the existing works in the domain, few works dealt with the data analysis. We addressed this issue by designing a decision support system based on fuzzy logic to take into consideration the imprecision of the data and the range of the referenced norms. The DSS can help the HP in making reliable assessments and improved medical decisions based on relevant parameter measurements. We showed the feasibility and the effectiveness of the proposed approach through the TUG test. The obtained results were consistent with the HP's expertise to $85 \%$. The developed DSS can objectively assess the fall risk in less than ten minutes and in an easy way. Furthermore, the solution is low-cost and non invasive 
Table 1: Rules of the inference system.

\begin{tabular}{|c|c|c|c|c|c|c|c|c|c|c|}
\hline \multirow{2}{*}{$\mathrm{N}^{\mathrm{O}}$} & & \multicolumn{7}{|c|}{ Inputs } & & \multirow{2}{*}{ Output } \\
\hline & & step length & step frequency & gait duration & gait velocity & hip AROM & Knee AROM & Ankle AROM & & \\
\hline$P_{1}$ & $\overline{\mathrm{IF}}$ & correct & correct & correct & correct & correct & correct & correct & THEN & Low risk \\
\hline$\overline{P_{2}}$ & $\overline{\mathrm{IF}}$ & low & low & high & low & low & low & & THEN & High risk \\
\hline$P_{3}$ & IF & low & correct & correct & correct & low & & & THEN & Moderate risk \\
\hline$P_{4}$ & IF & low & high & high & & low & & & THEN & Risk \\
\hline$P_{5}$ & IF & correct & low & & low & low & & & THEN & Moderate risk \\
\hline$P_{6}$ & IF & moderate & low & & low & & correct & bad & THEN & Risk \\
\hline$P_{7}$ & $\overline{\mathrm{IF}}$ & moderate & correct & & moderate & & & & THEN & Moderate risk \\
\hline
\end{tabular}

for the patient.

The next step is to obtain data from the 3 tests and to take into account the strength and the static balance to enhance the fall risk estimation. The HP's feedback will be crucial for the DSS refinement and for the improvement of the DSS efficiency.

\section{ACKNOWLEDGMENTS}

This work was supported by a Marie Curie Action, FP7PEOPLE-2012-IAPP, KINOPTIM project, grant nb 324491.

\section{REFERENCES}

[1] C. Barelle, E. Courtial, and al. Tele-monitoring and diagnostic for fall prevention : The kinoptim concept. In IEEE-EMBS Int. Conference on Biomedical and Health Informatics, June 2014.

[2] S. Buatois and al. A simple clinical scale to stratify risk of recurrent falls in community-dwelling adults aged 65 years and older. Physical Therapy, 90(4):550-560, 2010.

[3] Y. Charlon, E. Campo, D. Brulin, and A. Piau. Smart insole for measuring actimetry of frail people. In Med-e-tel 2015 : the International eHealth, Telemedicine and Health ICT Forum For Education, Networking and Business, April 2015.

[4] A. Derbel, N. Mansouri, Y. B. Jemaa, B. Emile, and S. Treuillet. Comparative study between spatio/temporal descriptors for pedestrians recognition by gait. In Lecture Notes in Computer Science, volume 7950, pages 35-42, 2013.

[5] B. R. Greene, A. O'Donovan, R. Romero-Ortuno, L. Cogan, C. N. Scanaill, and R. A. Kenny. Quantitative falls risk assessment using the timed up and go test. IEEE Trans. on Biomedical Engineering, 57(12):2918-2926, 2010.

[6] U. Nations. Health population ageing 2013. Report of the Department of Economic and Social Affairs, 2013.

[7] A. Pfister, A. M. West, S. Bronner, and J. A. Noah. Comparative abilities of microsoft kinect and vicon $3 \mathrm{~d}$ motion capture for gait analysis. Journal of Medical Engineering and Technology, 38(5):274-280, 2014.

[8] D. Podsiadlo and S. Richardson. The timed up and go: a test of basic functional mobility for frail elder persons. ournal of the American Geriatrics Society, 39(2):142-148, 1991.

[9] K. Sheehan, B. Greene, C. Cunningham, L. Crosby, and R. Kenny. Early identification of declining balance in higher functioning older adults, an inertial sensor based method. Gait and posture, 39(4):1034-1039, 2014.

[10] T.Oberg, A. Karsznia, and K. Oberg. Basic gait parameters : Reference data for normal subjects, 10-79 years of age. Journal of Rehabilitation Research and Development, 30(2):210-223, 1993.

[11] D. Trojaniello and al. Estimation of step-by-step spatio-temporal parameters of normal and impaired gait using shank-mounted magneto-inertial sensors. Journal of NeuroEngineering and Rehabilitation.

[12] WHO. Global report on falls prevention in older age. http://www.who.int/ageing/publications/Fallsprevention7March.pdf.

[13] D. Winter. The Biomechanics and Motor Control of Human Movement. John Wiley and Sons, New York, 1990.

[14] J. Yoo, M. Nixon, and C. Harris. Extracting gait signatures based on anatomical knowledge. In BMVA Workshop advancing biometric technologies, Cardiff 2002 . 